\title{
Deducing ink-transmittance spectra from reflectance and transmittance measurements of prints
}

\author{
Mathieu Hébert and Roger D. Hersch \\ Ecole Polytechnique Fédérale de Lausanne (EPFL), \\ School of Computer and Communication Sciences, \\ 1015 Lausanne, Switzerland.
}

\begin{abstract}
The color of prints is mainly determined by the light absorption of the inks deposited on top of paper. In order to predict the reflectance spectrum of prints, we use a spectral prediction model in which each ink is characterized by its spectral transmittance. In the present paper, we consider two classical reflectance prediction models: the Clapper-Yule model and the Williams-Clapper model. They rely on a same description of multiple reflection-transmission of light, but use a different description of the attenuation of light by the inks. In the Clapper-Yule model (non-orientational ink attenuation), the orientation of light traversing the ink is not taken into account. In the Williams-Clapper model, it is taken into account (orientational ink attenuation). In order to determine experimentally which of these two models is the more suitable for a given type of print, we propose a method using the reflectance and the transmittance of prints. We introduce a bimodal model, enabling spectral reflectance and transmittance predictions. Depending whether the direction of light into the ink is taken into account, we obtain a non-orientational bimodal model or an orientational bimodal model. Using these two models, we deduce the ink transmittance spectrum from various reflectance and transmittance measurements performed on a same print, and compare the different deduced spectra. The model which is the most adapted to the considered print is the one where the deduced spectra best match each other.
\end{abstract}

Keywords: Color reproduction, print, spectral prediction model, reflectance-transmittance model, Clapper-Yule model, Williams-Clapper model, ink transmittance.

\section{INTRODUCTION}

In color halftone printing, colors are formed by depositing on a support, e.g. a sheet of paper, color inks covering each one a given proportion of the support surface. One of the main challenges is the determination of the relationship between the surface coverages of the primary inks and the real color seen by an observer. We generally assume that the color perceived by an observer is fully determined by the spectrum of the light received by the observer's eye. This spectrum is the product of the spectrum of the light source with a spectral function characteristic of the print, i.e. its reflectance or transmittance spectrum. Hence, controlling the print colors implies that, given the surface coverage of the different inks, the print's reflectance or/and transmittance spectra can be predicted. For the prediction of reflectance and/or transmittance spectra, a model is used which includes the different optical phenomena taking place within the print (reflection, transmission, absorption, scattering etc.) as well as the physical interactions of inks and paper (penetration of inks within the support, etc.). The prediction model relies on parameters characterizing quantitatively the different phenomena. Its accuracy depends on the precision according to which the different physical and optical phenomena are described. The Clapper-Yule model ${ }^{1}$ was specially introduced for predicting the reflectance of halftone prints having sufficiently high halftone screen frequencies. Light is assumed to be attenuated by a same factor when crossing the ink layer, independently of its angular distribution (non-orientational model). Williams and Clapper ${ }^{2}$ proposed a slightly different model for photography, which is also suitable for paper sheets coated with a transparent coloring layer. In the Williams-Clapper model, as well as in its extension by Shore and Spoonhower ${ }^{3}$, the attenuation of light within the ink is computed according to Beer's law in respect to its angular distribution (orientational model).

M. Hébert and R.D. Hersch, IS\&T/SPIE Electronic Imaging Symposium, Color Imaging XII: Processing, Hardcopy and Applications, SPIE Vol. 6493, pp. 649314-1-13. 
In the present paper, we analyze whether the Clapper-Yule model with its non-orientational ink attenuation or the Williams-Clapper model with its orientational ink attenuation is preferable for predicting the reflectance spectrum of solid ink patches (100\% coverage of one primary ink). We propose a method relying on the transmittance of prints. In addition to the print reflectance measurement, we can perform two transmittance measurements: one measurement with the ink being at the source side, the other with the ink being at the detector side. Since we can use each of these three measurements to deduce the spectral ink transmittance, we obtain three spectra which should be identical if the model is correct. As reflectance and transmittance prediction model, we use a bimodal model in which the attenuation of light within the ink can be modeled either like in the Clapper-Yule model or like in the Williams-Clapper model. We thus obtain a "non-orientational bimodal model" and an "orientational bimodal model". By comparing the three ink transmittance spectra deduced using each of the two models, we can easily determine the model for which the three deduced spectra best match each other.

In Section 2, we present in detail the Clapper-Yule model and the Williams-Clapper model. The non-orientational and the orientational bimodal models are presented in Section 3. For each version of the bimodal model, we develop the equations that should be used to deduce the ink transmittance spectra from the print reflectance and from the two print transmittances. We use these equations in Section 4 with offset inks printed on white PVC. We explain how to determine whether the non-orientational approach (Clapper-Yule model) or the orientational approach (Williams-Clapper model) is the most adapted to the considered print, and draw the conclusions in Section 5.

\section{REFLECTANCE-ONLY MODEL}

According to reflectance models such as the Clapper-Yule model or the Williams-Clapper model, the light being reflected by a print is subject to multiple reflections and transmissions between three elements: a paper background comprising the paper bulk and its backing paper-air interface, an ink layer, and the ink-air interface. According to these models, the paper background is a Lambertian reflector, whose reflectance $\rho_{B}$ is independent of the angular distribution of the received light ${ }^{4}$. The light that it reflects is perfectly diffused, i.e. Lambertian. The ink layer has the same refractive index $n$ as the paper (typically $n=1.5$ ), thereby preventing Fresnel reflections or transmissions at the paper-ink boundary. The ink-air interface is perfectly smooth.

The ink layer and the ink-air interface may be grouped together, forming a single element called a colored interface ${ }^{5}$. If the paper is not printed, the colored interface corresponds to the paper-air interface alone. A print is thus composed of its paper background and its colored interface. The reflectance model describes the multiple reflections of light between these two elements.

\subsection{Multiple reflection-transmission of light into the print}

A light source illuminates the print with a certain angular distribution (e.g. monodirectional or Lambertian). Depending on this angular distribution, a fraction $T_{i n}$ of the incident light crosses the colored interface. Then, it penetrates into the paper background where it is diffused and partially absorbed (Fig. 1). A fraction $\rho_{B}$, called the background reflectance, is reflected back to the colored interface, where it is subsequently split into two components: a fraction $r$ is internally reflected by the colored interface and returns back into the paper background; a fraction $T_{e x}$ exits the print and is captured by the detector according to the measuring geometry (e.g. a radiance detector capturing light at a given angle, or an integrating sphere capturing all the reflected light). Light is thus subject to multiple reflections between the background and the colored interface, with a fraction of light exiting the print and being captured by the detector at each cycle of reflections.

By summing the fractions of light exiting the print at each cycle, we obtain the total amount of light reflected by the print, which also corresponds to the print global reflectance $R$

$$
R=T_{i n}\left[\rho_{B}+\rho_{B} r \rho_{B}+\rho_{B}\left(r \rho_{B}\right)^{2}+\ldots\right] T_{e x}
$$




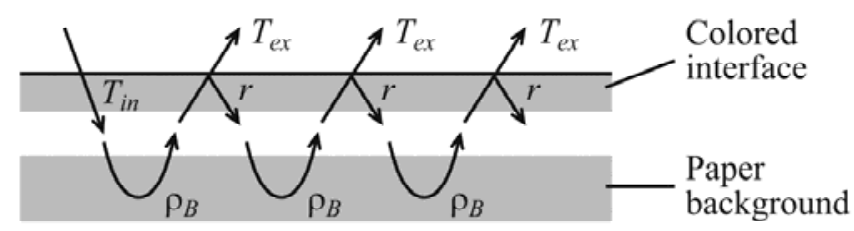

Fig. 1. Multiple reflections between the colored interface and the paper background.

The infinite sum is a geometric series converging towards $\rho_{B} /\left(1-r \rho_{B}\right)$. Therefore, the print global reflectance is expressed by

$$
R=T_{i n} T_{e x} \frac{\rho_{B}}{1-r \rho_{B}}
$$

In Eq. (2), the penetration term $T_{i n}$, the exit term $T_{e x}$ and the internal reflectance $r$ are characteristic of the colored interface. Their expression need to be calculated according to the basic optical laws describing the behavior of flat interfaces (Snell's laws, Fresnel's formulae) and according to a model for the attenuation of the light in the ink.

In the following sections, we use the same notations as in Ref. 5 concerning Fresnel coefficients. Let us consider, for example, a flat interface between paper (medium 1 having a typical refractive index $n_{1}=1.5$ ) and air (medium 0 having the refractive index $n_{0}=1$ ). For directional light coming from air at angle $\theta_{0}$, the Fresnel reflection factor is noted $R_{01}\left(\theta_{0}\right)$ and is given by Fresnel's formulae ${ }^{6}$. For directional light incident at angle $\theta_{1}$ at the paper side, the Fresnel transmission factor is noted $T_{10}\left(\theta_{1}\right)$. For Lambertian incident light, the interface reflectance, resp. transmittance, is obtained by integrating angularly the Fresnel reflection factor, resp. transmission factor ${ }^{7}$.

\subsection{Characterization of the paper background}

In order to compute the paper background reflectance $\rho_{B}$, we take an unprinted paper sheet. On top of the paper background, we have the flat paper-air interface (colored interface). Let us express parameters $r, T_{i n}$, and $T_{e x}$.

The internal reflectance $r$ is the fraction of Lambertian light coming from the paper which is reflected by the paper-air interface. It corresponds to the Lambertian reflectance of flat interfaces, noted $r_{10}$ and defined as ${ }^{4,8}$

$$
r_{10}=\int_{\theta=0}^{\pi / 2} R_{10}(\theta) \sin 2 \theta d \theta
$$

Table 1. Attenuation of incident light traversing the air-paper interface according to the geometry of illumination ${ }^{5}$

\begin{tabular}{|l|l|}
\hline Directional incident light at angle $\theta$ & $T_{i n}^{p}=T_{01}(\theta)$ \\
\hline Directional incident light at $45^{\circ}$ with $n=1.5$ & $T_{i n}^{p}=0.95$ \\
\hline Lambertian incident light & $T_{i n}^{p}=t_{01}$ \\
\hline Lambertian incident light with $n=1.5$ & $T_{i n}^{p}=0.90$ \\
\hline
\end{tabular}

Table 2. Attenuation of emerging light reaching the light detector according to the measuring geometry ${ }^{5}$

\begin{tabular}{|l|l|}
\hline Radiance detector at angle $\theta^{\prime}$ & $T_{e x}^{p}=T_{01}\left(\theta^{\prime}\right) / n^{2}$ \\
\hline Radiance detector at $0^{\circ}$ with $n=1.5$ & $T_{e x}^{p}=0.96$ \\
\hline Integrating sphere & $T_{e x}^{p}=t_{10}$ \\
\hline
\end{tabular}




\begin{tabular}{|l|l|}
\hline Integrating sphere with $n=1.5$ & $T_{e x}^{p}=0.40$ \\
\hline
\end{tabular}

Penetration term $T_{i n}$ and exit term $T_{e x}$ depend respectively on the illumination geometry and on the measuring geometry. Expressions for $T_{\text {in }}$ are presented in Table 1 for directional and for Lambertian incident light. Expressions for $T_{e x}$ are given in Table 2 for a radiance detector or for an integrating sphere as light collector. We refer to these special expressions in the next sections respectively as $T_{i n}^{p}$ and $T_{e x}^{p}$.

For example, let us consider directional light incident at $\theta=45^{\circ}$, a radiance detector placed at $\theta^{\prime}=0^{\circ}$, and a paper refractive index $n=1.5$. The relative index of refraction of the paper-air interface is therefore $n=n_{1} / n_{0}=1.5$. The corresponding values for $T_{i n}^{p}$ and $T_{e x}^{p}$ are given in Table 1 and Table 2 respectively. The internal reflectance of the paper-air interface, for a paper refractive index of 1.5, is $r_{10}=0.6$. By inserting these values for $r$, $T_{i n}$, and $T_{e x}$ into Eq. (2), we obtain the following expression for the reflectance of an unprinted paper sheet

$$
R=T_{01}(\theta) \frac{T_{01}\left(\theta^{\prime}\right) \quad \rho_{B}}{n^{2} 1-r_{10} \rho_{B}}=\frac{0.4 \rho_{B}}{1-0.6 \rho_{B}}
$$

By measuring the reflectance spectrum $R(\lambda)$ of the unprinted paper, it is possible to deduce numerically, wavelength by wavelength, the reflectance spectrum of the paper background $\rho_{B}(\lambda)$.

\subsection{Clapper-Yule model}

We now consider the same paper sheet as above, coated with a homogenous ink layer. On top of the paper background, whose reflectance $\rho_{B}$ has already been determined, we have a colored interface formed by the ink layer and the ink-air interface. Since the ink and the paper have the same refractive index $n_{1}$, the ink-air interface has the same optical properties as the paper-air interface. We have therefore the same Fresnel terms as for the unprinted paper.

According to the Clapper-Yule model, light is attenuated by a same factor $\hat{t}$ each time it crosses the ink layer. Factor $\hat{t}$, called the invariant ink transmittance of the ink layer, is independent of the angular distribution of light. Let us expand the penetration term, the exit term and the internal reflectance, noted respectively $T_{i n}(\hat{t}), T_{e x}(\hat{t})$ and $r(\hat{t})$, according to the Clapper-Yule model.

When light penetrates the print, it first crosses the ink-air interface (transmittance $T_{i n}^{p}$, like for the paper-air interface, depending on the angular distribution of the light source) and then the ink layer (attenuation factor $\hat{t}$ ). Therefore, the penetration term is

$$
T_{i n}(\hat{t})=T_{i n}^{p} \hat{t}
$$

Likewise, the exiting light captured by the detector crosses the ink layer (attenuation factor $\hat{t}$ ) and the ink-air interface (transmittance $T_{e x}^{p}$, depending on the measuring geometry). The exit term is

$$
T_{e x}(\hat{t})=T_{e x}^{p} \hat{t}
$$

The internal reflectance of the colored interface accounts for the transmission of the Lambertian light issued from the paper background across the ink layer (attenuation factor $\hat{t}$ ), its internal reflection at the ink-air interface (reflectance $r_{10}$ given by Eq. (3)), and its second transmission across the ink layer (attenuation factor $\hat{t}$ again). Therefore, the internal reflectance is

$$
r(\hat{t})=r_{10} \hat{t}^{2}
$$

For example, we have a print whose refractive index is $n=1.5$. It is illuminated at $\theta=45^{\circ}$ and light is collected by a radiance detector at $\theta^{\prime}=0^{\circ}$. According to Tables 1 and 2, we have $T_{i n}^{p}=T_{01}\left(45^{\circ}\right)=0.95$ and $T_{e x}^{p}=T_{01}\left(0^{\circ}\right) / n^{2}=0.43$. By inserting Eqs. (5), (6) and (7) into Eq. (2), we obtain the following expression for the print reflectance according to the Clapper-Yule model 


$$
R=T_{01}(\theta) \frac{T_{01}\left(\theta^{\prime}\right) \quad \rho_{B} \hat{t}^{2}}{n^{2} \quad 1-r_{10} \rho_{B} \hat{t}^{2}}=\frac{0.4 \rho_{B} \hat{t}^{2}}{1-0.6 \rho_{B} \hat{t}^{2}}
$$

Note that Eq. (8) corresponds to the particular case of the classical Clapper-Yule reflectance model where $a=1$, with $a$ being the fraction of the paper surface which is covered by the ink $\mathrm{k}^{1,4}$

$$
R=T_{01}(\theta) \frac{T_{01}\left(\theta^{\prime}\right) \quad \rho_{B}(1-a+a \hat{t})^{2}}{n^{2} \quad 1-r_{10} \rho_{B}\left(1-a+a \hat{t}^{2}\right)}
$$

The paper background reflectance $\rho_{B}$ has already been computed from Eq. (4). We can measure the print reflectance spectrum $R(\lambda)$ and deduce numerically, wavelength by wavelength, the mean attenuation factor $\tilde{t}(\lambda)$ using Eq. (8).

\subsection{Williams-Clapper model}

In the Williams-Clapper model, the ink layer is explicitly assumed to be transparent. According to Beer's law ${ }^{9}$, light is attenuated within the ink layer as a function of the length of the traversed path. The length of the traversed path is a function of its orientation. When light crosses the layer perpendicularly, it is attenuated by a factor $t$ called the normal transmittance of the ink layer. When it is incident at a nonzero angle $\theta$, it traverses a path of relative length $1 / \cos \theta$ within the ink layer and is, according to Beer's law, attenuated by a factor $t^{1 / \cos \theta}$.

The colored interface is composed of the transparent ink layer and of the flat ink-air interface. Neither the ink layer or the ink-air interface scatter light. Therefore, the travel of light within the colored interface and its global attenuation are determined by the angle of incidence of light. When the incident light is directional, the angles of reflection and transmission are determined according to Snell's laws. The proportion of reflection and transmission are given by angular functions, depending only on the angle of incidence, derived from Fresnel's formulae and Beer's law. When the incident light is diffuse, the reflectance and the transmittance of the colored interface are obtained by integrating these angular functions over the range of incidence. This is called the principle of directionality. The Williams-Clapper model is therefore an "orientational model".

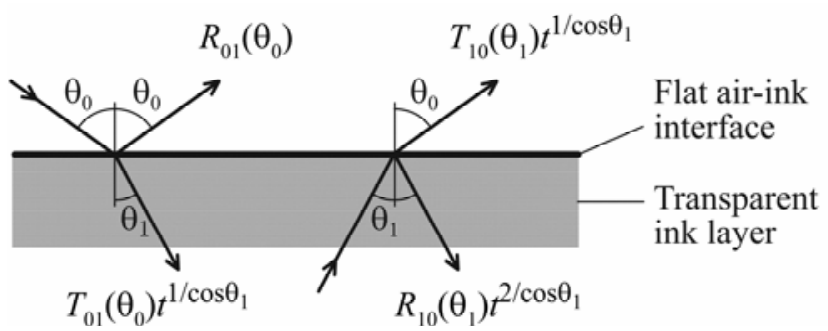

Fig. 2: Reflection and transmission of light across the colored interface according to the Williams-Clapper model. Angles $\theta_{0}$ and $\theta_{1}$ are related according to Snell's laws, i.e. $\sin \theta_{0}=n \sin \theta_{1}$.

In order to illustrate the principle of directionality, let us consider the transmission of light coming from air through the colored interface. If the incident light is directional at angle $\theta_{0}$, a fraction $T_{01}\left(\theta_{0}\right) t^{1 / \cos \theta_{1}}$ is transmitted (see Fig. 2), which is a function of the incident angle $\theta_{0}$ since $\theta_{1}=\arcsin \left(\sin \theta_{0} / n\right)$. If the incident light is Lambertian, we integrate this function over the hemisphere and obtain the fraction $p(t)$ of Lambertian incident light which is transmitted across the colored interface

$$
p(t)=\int_{\theta_{0}=0}^{\pi / 2} T_{01}\left(\theta_{0}\right) t^{1 / \sqrt{1-\left(\sin \theta_{0} / n\right)^{2}}} \sin 2 \theta_{0} d \theta_{0}
$$

Let us now express the internal reflectance $r$ o the colored interface. It is the fraction of Lambertian incident light emitted by the paper background which is reflected by the colored interface. Since light travels twice through the ink layer, the 
reflection of directional light by the colored interface at the paper side is characterized by the angular function $t^{2 / \cos \theta_{1}} R_{10}\left(\theta_{1}\right)$ (see Fig. 2). For Lambertian incident light, we integrate this function angularly over the hemisphere. We note $r(t)$ the resulting internal reflectance, expressed according to the Williams-Clapper model

$$
r(t)=\int_{\theta_{1}=0}^{\pi / 2} t^{2 / \cos \theta_{1}} R_{10}\left(\theta_{1}\right) \sin 2 \theta_{1} d \theta_{1}
$$

Function $r(t)$ has been tabulated ${ }^{4}$ as a function of the ink normal transmittance $t$ for $n=1.5$. The penetration term and the exit term, noted respectively $T_{i n}(t)$ and $T_{e x}(t)$, are presented in Tables 3 and 4 depending on the illumination and measuring geometries. Note that the expressions presented in Tables 3 and 4 were not presented in the original Williams and Clapper's paper, but in its extensions proposed by Shore and Spoonhower ${ }^{3}$, or Hébert and Hersch ${ }^{4,5}$.

Table 3. Penetration $T_{\text {in }}(t)$ of the incident light across the colored interface, depending on the geometry of illumination

\begin{tabular}{|l|l|}
\hline Directional incident light at angle $\theta_{0}$ & $T_{\text {in }}(t)=T_{01}\left(\theta_{0}\right) t^{1 / \sqrt{1-\left(\sin \theta_{0} / n\right)^{2}}}$ \\
\hline Directional incident light at $45^{\circ}$ with $n=1.5$ & $T_{\text {in }}(t)=0.95 t^{1.13}$ \\
\hline Lambertian incident light & $T_{\text {in }}(t)=p(t)[$ Eq. (10)] \\
\hline Lambertian incident light with $n=1.5$ & $T_{\text {in }}(t) \approx 0.90 t^{1.13}$ \\
\hline
\end{tabular}

Table 4. Exit $T_{e x}(t)$ across the colored interface of the light captured by the detector, depending on the measuring geometry

\begin{tabular}{|l|l|}
\hline Radiance detector at angle $\theta$ & $T_{e x}(t)=T_{01}\left(\theta_{0}\right) t^{1 / \sqrt{1-\left(\sin \theta_{0} / n\right)^{2}}} / n^{2}$ \\
\hline Radiance detector at $0^{\circ}$ with $n=1.5$ & $T_{e x}(t)=0.96 t$ \\
\hline Integrating sphere & $T_{e x}(t)=\int_{\theta_{1}=0}^{\pi / 2} T_{10}\left(\theta_{1}\right) t^{1 / \cos \theta_{1}} \sin 2 \theta_{1} d \theta_{1}$ \\
\hline Integrating sphere with $n=1.5$ & $T_{e x}(t) \approx 0.40 t^{1.13}$ \\
\hline
\end{tabular}

For example, let us consider directional incident light at angle $\theta$ and a radiance detector at angle $\theta^{\prime}$. According to the Williams-Clapper model, the reflectance of a print with a ink solid layer having a normal transmittance $t$ is expressed as ${ }^{3}$

$$
R=T_{01}(\theta) \frac{T_{01}\left(\theta^{\prime}\right)}{n^{2}} \cdot t^{1 / \sqrt{1-(\sin \theta / n)^{2}}} \cdot t^{1 / \sqrt{1-\left(\sin \theta^{\prime} / n\right)^{2}}} \cdot \frac{\rho_{B}}{1-r_{1}(t) \rho_{B}}
$$

Since the paper background reflectance $\rho_{B}(\lambda)$ has already been computed, the ink normal transmittance spectrum $t(\lambda)$ can be deduced from the measured print reflectance spectrum $R(\lambda)$ using Eq. (12).

\subsection{Extending the reflectance only models}

We have presented two versions of the reflectance-only model, in which the attenuation of light within the ink is modeled either with (Clapper-Yule) or without (Williams-Clapper) consideration of its angular distribution. In practice, it is often difficult to know the model which should be used. We need an experimental method permitting to determine which approach is the more consistent with the optical phenomena taking place within the ink.

Reflectance-only models do not offer enough measurements compared with the number of parameters. We can only measure the reflectance of the unprinted paper, which is used to compute the paper background reflectance, and the reflectance of the print, which is used to compute the ink transmittance. Since we have no other measurement, we cannot verify the computed ink transmittance. We can measure the transmittance of the unprinted paper and the transmittance of the print, which can be used for verifying the deduced ink transmittance spectra. Let us now present the bimodal model which makes use of both the reflectance and the transmittance of prints. 


\section{BIMODAL MODEL}

According to the bimodal model, the print is composed by a central layer of paper bulk bordered by two colored interfaces. The paper is a strongly scattering layer. It may be nonsymmetric, i.e. have an intrinsic reflectance $\rho_{1}$ at one side and a different intrinsic reflectance $\rho_{2}$ at the other side. Its transmittance $\tau$ is side-independent according to the principle of nonpolarity for the transmittance of strongly scattering layers ${ }^{10}$. Each colored interface is either the paper-air interface without ink, or an ink layer together with its ink-air interface. For the colored interfaces, we have: a penetration term $T_{i n}$, an exit term $T_{e x}$ and the internal reflectance of the colored interfaces, noted $r_{1}$ for the recto colored interface (i.e. the one which is in front of the light source) and $r_{2}$ of the verso colored interface (Fig. 3).

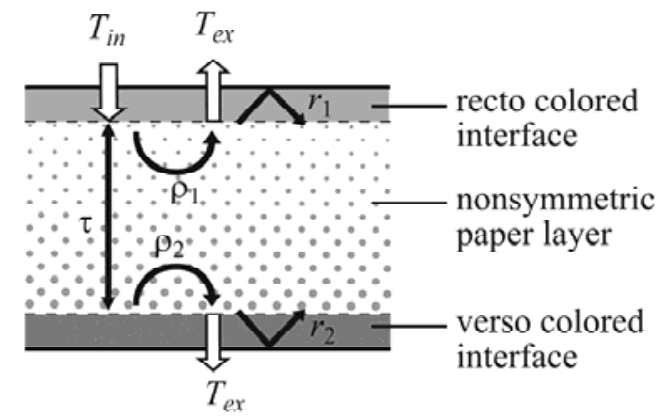

Fig. 3: Print composed of a central nonsymmetric paper layer, having intrinsic parameters $\rho_{1}, \rho_{2}$ and $\tau$, bordered by colored interfaces having respective internal reflectances $r_{1}$ and $r_{2}$. The penetration term $T_{i n}$ and the exit term $T_{e x}$ depend on the measuring geometry.

In previous contributions ${ }^{11,12,4}$, the following expressions are proposed for the print reflectance

$$
R=T_{i n} T_{e x} \frac{\rho_{1}-r_{2}\left(\rho_{1} \rho_{2}-\tau^{2}\right)}{\left(1-r_{1} \rho_{1}\right)\left(1-r_{2} \rho_{2}\right)-r_{1} r_{2} \tau^{2}}
$$

and for the print transmittance

$$
T=T_{i n} T_{e x} \frac{\tau}{\left(1-r_{1} \rho_{1}\right)\left(1-r_{2} \rho_{2}\right)-r_{1} r_{2} \tau^{2}}
$$

Note that the paper background considered in the reflectance-only model corresponds to the composition of the central paper layer, characterized by its intrinsic parameters $\rho_{1}, \rho_{2}$ and $\tau$, and of the verso colored interface, having a reflectance $r_{2}$. If we describe the multiple reflections between the paper layer and the verso colored interface, we can express the background reflectance $\rho_{B}$ as a function of $\rho_{1}, \rho_{2}$ and $\tau$ and $r_{2}$ (see Fig. 4).

$$
\rho_{B}=\rho_{1}+\tau\left(1+r_{2} \rho_{2}+r_{2}^{2} \rho_{2}^{2}+\ldots\right) r_{2} \tau=\rho_{1}+\frac{r_{2} \tau^{2}}{1-r_{2} \rho_{2}}
$$

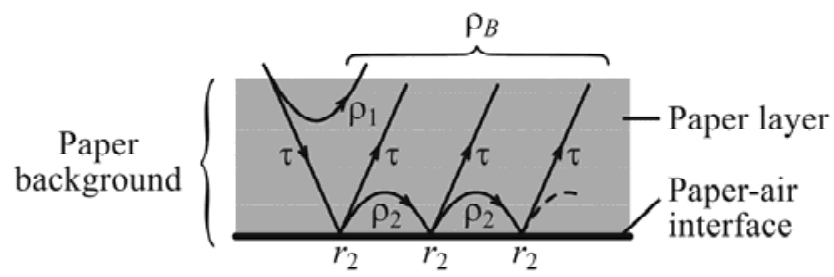

Fig. 4. The paper background reflectance $\rho_{B}$ includes the multiple reflections taking place between the paper layer, having the intrinsic parameters $\rho_{1}, \rho_{2}$ and $\tau$, and its backing interface whose reflectance is $r_{2}$. 
By replacing $\rho_{B}$ with its expression (15) into Eq. (2), we retrieve exactly Eq. (14). This shows that the reflectance-only model and the bimodal model are equivalent compatible reflectance prediction models ${ }^{4}$.

In the next sections, we describe the expanded expressions for parameters $T_{i n}, T_{e x}, r_{1}$ and $r_{2}$ either by considering an unprinted paper sheet (Section 3.1), a print where the attenuation of light into the ink is modeled like in the Clapper-Yule model (Section 3.2) or a print where the attenuation of light into the ink is modeled like in the Williams-Clapper model (Section 3.3).

In order to reduce the number of equations, we consider a single measuring geometry for the reflectance mode, and a single geometry for the transmittance mode. In reflectance mode, directional light is incident at $45^{\circ}$ and light is captured by a radiance detector at $0^{\circ}$ (classical $45^{\circ} / 0^{\circ}$ geometry). In transmittance mode, the incident light is Lambertian and the transmitted light is also captured by a radiance detector at $0^{\circ}$ (diffuse $/ 0^{\circ}$ geometry).

\subsection{Characterization of the paper layer}

The paper intrinsic parameters $\rho_{1}, \rho_{2}$ and $\tau$ are deduced from three different spectral measurements performed on the unprinted paper sheet. We measure the sheet reflectance $R_{1}$ using a $45^{\circ} / 0^{\circ}$ geometry and the sheet transmittance $T$ using a diffuse $/ 0^{\circ}$ geometry. Then, we flip the sheet (the recto takes the place of the verso and conversely) and measure its reflectance $R_{2}$, using again the $45^{\circ} / 0^{\circ}$ geometry.

The paper sheet is composed of a central paper layer bordered by identical paper-air interfaces. Their internal reflectance at the paper side is $r_{10}$, given by Eq. (3). Hence, we have

$$
r_{1}=r_{2}=r_{10}
$$

Terms $T_{i n}$ and $T_{e x}$ depend on the measuring geometry selected in reflectance and transmittance modes. Since the two modes use a radiance detector at $0^{\circ}$, the exit term $T_{e x}$ is (see Table 2)

$$
T_{e x}=T_{10}\left(0^{\circ}\right) / n^{2}
$$

In reflectance mode, light is incident at $45^{\circ}$ and the penetration term is

$$
T_{\text {in }}=T_{01}\left(45^{\circ}\right)
$$

In transmittance mode, the incident light is Lambertian, which yields the penetration term (see Table 1)

$$
T_{\text {in }}=t_{01}=\int_{\theta_{0}=0}^{\pi / 2} T_{01}\left(\theta_{0}\right) \sin 2 \theta_{0} d \theta_{0}
$$

By inserting expressions (16), (17) and (18) into Eq. (13), we obtain the following expression for the paper sheet reflectance

$$
R_{1}=\frac{T_{01}\left(45^{\circ}\right) T_{10}\left(0^{\circ}\right)}{n^{2}} \cdot \frac{\rho_{1}-r_{10}\left[\rho_{1} \rho_{2}-\tau^{2}\right]}{\left(1-r_{10} \rho_{1}\right)\left(1-r_{10} \rho_{2}\right)-r_{10}^{2} \tau^{2}}
$$

and by inserting Eqs. (16), (17) and (19) into Eq. (14), we obtain the following expression for the sheet transmittance

$$
T=\frac{t_{01} T_{10}\left(0^{\circ}\right)}{n^{2}} \cdot \frac{\rho_{1}-r_{10}\left[\rho_{1} \rho_{2}-\tau^{2}\right]}{\left(1-r_{10} \rho_{1}\right)\left(1-r_{10} \rho_{2}\right)-r_{10}^{2} \tau^{2}}
$$

The flipped sheet reflectance $R_{2}$ is expressed as in Eq. (20) by having $\rho_{1}$ and $\rho_{2}$ replacing each other

$$
R_{2}=\frac{T_{01}\left(45^{\circ}\right) T_{10}\left(0^{\circ}\right)}{n^{2}} \cdot \frac{\rho_{2}-r_{10}\left[\rho_{2} \rho_{1}-\tau^{2}\right]}{\left(1-r_{10} \rho_{1}\right)\left(1-r_{10} \rho_{2}\right)-r_{10}^{2} \tau^{2}}
$$


In Eqs. (20), (21) and (22), only parameters $\rho_{1}, \rho_{2}$ and $\tau$ are unknown. The other terms are constants determined by Fresnel formulae as functions of the paper-air relative refractive index $n$. These three equations permit to deduce numerically, wavelength by wavelength, the three intrinsic parameters $\rho_{1}, \rho_{2}$ and $\tau$ of paper.

If the paper layer is symmetrical, its two internal reflectances are equal, i.e. $\rho_{1}=\rho_{2}=\rho$. For deducing the paper intrinsic reflectance $\rho$ and transmittance $\tau$, we just have to measure the sheet reflectance $R$ and the sheet transmittance $T$, given respectively by

$$
R=\frac{T_{01}\left(45^{\circ}\right) T_{10}\left(0^{\circ}\right)}{n^{2}} \cdot \frac{\rho-r_{10}\left[\rho^{2}-\tau^{2}\right]}{\left(1-r_{10} \rho\right)^{2}-r_{10}^{2} \tau^{2}}
$$

and by

$$
T=\frac{t_{01} T_{10}\left(0^{\circ}\right)}{n^{2}} \cdot \frac{\rho_{1}-r_{10}\left[\rho^{2}-\tau^{2}\right]}{\left(1-r_{10} \rho\right)^{2}-r_{10}^{2} \tau^{2}}
$$

Once the intrinsic parameters of paper are computed, the paper is characterized. Then, we can consider an ink layer coated on the paper sheet.

\subsection{Non-orientational bimodal model}

The solid ink patch is assumed to be composed of the central paper layer characterized in Section 3.1, bordered at one side by a colored interface composed of the ink layer and the ink-air interface, and bordered at the other side by the paper-air interface. Like in the Clapper-Yule model, we assume that light is attenuated by an invariant factor $\tilde{t}$ each time it crosses the ink layer, independently on its angular distribution. Terms $T_{i n}$ and $T_{e x}$ depend on the measuring geometries used in reflectance and transmittance modes. Let us express them according to the geometries selected for the next three measurements.

First, we measure the print reflectance, using the $45^{\circ} / 0^{\circ}$ geometry. The penetration term $T_{\text {in }}$ includes the transmission of the incident light across the ink-air interface and across the ink layer

$$
T_{\text {in }}=T_{01}\left(45^{\circ}\right) \hat{t}
$$

The exit term $T_{e x}$ includes the transmission of the radiance captured by the radiance detector across the ink layer and the ink-air interface

$$
T_{e x}=\frac{T_{01}\left(0^{\circ}\right) \hat{t}}{n^{2}}
$$

Since the recto colored interface contains the ink layer and since the verso colored interface is the paper-air interface alone, their respective internal reflectance $r_{1}$ and $r_{2}$ are given respectively by Eqs. (7) and (3)

$$
r_{1}=r_{10} \hat{t}^{2} \text { and } r_{2}=r_{10}
$$

Let us insert into Eq. (13) the expressions for $T_{i n}, T_{e x}, r_{1}$ and $r_{2}$ given respectively by Eqs. (25), (26) and (27). The print reflectance $R$ obtained according to the non-orientational bimodal model is

$$
R=\frac{T_{01}\left(45^{\circ}\right) T_{10}\left(0^{\circ}\right)}{n^{2}} \cdot \hat{t}^{2} \cdot \frac{\rho_{1}-r_{10}\left[\rho_{1} \rho_{2}-\tau^{2}\right]}{\left[1-r_{10} \hat{t}^{2} \rho_{1}\right]\left[1-r_{10} \rho_{2}\right]-r_{10}^{2} \hat{t}^{2} \tau^{2}}
$$

Then, we measure the transmittance of the print using a diffuse $/ 0^{\circ}$ geometry. The ink is positioned at the side of the Lambertian light source. The penetration term $T_{\text {in }}$ includes the transmission of the Lambertian incident light across the ink-air interface (transmittance $t_{01}$ given by Eq. (19)) and across the ink layer 


$$
T_{\text {in }}=t_{01} \hat{t}=\hat{t} \int_{\theta=0}^{\pi / 2} T_{01}(\theta) \sin 2 \theta d \theta
$$

The exit term $T_{e x}$ includes the transmission of the radiance captured by the radiance detector across the paper-air interface at the verso side (see Table 2)

$$
T_{e x}=\frac{T_{10}\left(0^{\circ}\right)}{n^{2}}
$$

Once again, the recto colored interface contains the ink layer $\left(r_{1}=r_{10} \hat{t}^{2}\right)$ and the verso colored interface is the paper-air interface $\left(r_{2}=r_{10}\right)$. By inserting into Eq. (14) parameters $T_{i n}, T_{e x}, r_{1}$ and $r_{2}$ given by Eqs. (27), (29) and (30), we obtain the following expression for the print transmittance $T$ according to the non-orientational bimodal model

$$
T_{1}=\frac{t_{10} T_{10}\left(0^{\circ}\right)}{n^{2}} \cdot \hat{t} \cdot \frac{\tau}{\left[1-r_{10} \hat{t}^{2} \rho_{1}\right]\left[1-r_{10} \rho_{2}\right]-r_{10}^{2} \hat{t}^{2} \tau^{2}}
$$

Third, we flip the print and measure again its transmittance. The ink is now at the detector side. Therefore, we have

$$
T_{i n}=t_{01}, \quad T_{e x}=\frac{T_{10}\left(0^{\circ}\right)}{n^{2}} \hat{t}, \quad r_{1}=r_{10} \quad \text { and } \quad r_{2}=r_{10} \hat{t}^{2}
$$

Since when flipping the print we also reverse the paper layer, internal reflectances $\rho_{1}$ and $\rho_{2}$ must be mutually exchanged. By inserting Eqs. (32) into Eq. (14) and replacing $\rho_{1}$ and $\rho_{2}$ with each other, the flipped print transmittance according to the non-orientational bimodal model is

$$
T_{2}=\frac{t_{10} T_{10}\left(0^{\circ}\right)}{n^{2}} \cdot \hat{t} \cdot \frac{\tau}{\left[1-r_{10} \rho_{2}\right]\left[1-r_{10} \hat{t}^{2} \rho_{1}\right]-r_{10}^{2} \hat{t}^{2} \tau^{2}}
$$

We can verify that $T_{1}=T_{2}$, i.e., according to the non-orientational bimodal model, the print transmittance is independent of the side of illumination. In practice, we may easily verify whether the print has the same transmittance when the ink is placed in front of the source or in front of the detector. If the print transmittance varies, we can conclude that the directionality of light into the ink layer should be taken into account.

\subsection{Orientational bimodal model}

Let us now consider that the ink is transparent and let us describe the attenuation of light within the ink like in the Williams-Clapper model. We perform the same reflectance and transmittance measurements as in Section 3.2, using the same measuring geometries in reflectance and transmittance modes.

The transparent ink layer has a normal transmittance $t$. Parameters $T_{i n}, T_{e x}, r_{1}$ and $r_{2}$ are computed in respect to the principle of directionality of the colored interface (see Section 2.4). The colored interface containing the ink layer has the internal reflectance $r(t)$ defined in Eq. (11). The other colored interface, being the paper-air interface, has the internal reflectance $r_{10}$ given by Eq. (3). Each internal reflectance correspond either to $r_{1}$ or to $r_{2}$ according to the side which is illuminated by the light source (see Table 5). Terms $T_{i n}$ and $T_{e x}$ account for the angle-dependent transmission of light across the ink layer depending on the measuring geometry. Their expressions are presented in Table 5 for each of the three considered measurements.

The print reflectance according to the orientational bimodal model is given by Eq. (13), with $T_{i n}, T_{e x}, r_{1}$ and $r_{2}$ being detailed in the left column of Table 5

$$
R=\frac{T_{01}\left(45^{\circ}\right) T_{01}\left(0^{\circ}\right)}{n^{2}} t^{1+1 / \sqrt{1-\left(\sin 45^{\circ} / n\right)^{2}}} \frac{\rho_{1}-r_{10}\left[\rho_{1} \rho_{2}-\tau^{2}\right]}{\left[1-r(t) \rho_{1}\right]\left[1-r_{10} \rho_{2}\right]-r(t) r_{10} \tau^{2}}
$$


Table 5. Expressions for $T_{e x}$ and $T_{e x}$ in reflectance and transmittance modes according to the orientational bimodal model ${ }^{4}$

\begin{tabular}{|c|c|c|c|}
\hline & $\begin{array}{l}\text { Reflectance measurement } \\
45^{\circ} / 0^{\circ} \text { geometry }\end{array}$ & $\begin{array}{l}\text { Transmittance measurement } \\
\text { Diffuse } / 0^{\circ} \text { geometry } \\
\text { Ink at the source side }\end{array}$ & $\begin{array}{l}\text { Transmittance measurement } \\
\text { Diffuse } / 0^{\circ} \text { geometry } \\
\text { Ink at the detector side }\end{array}$ \\
\hline$T_{i n}$ & $T_{01}\left(45^{\circ}\right) t^{1 / \sqrt{1-\left(\sin 45^{\circ} / n\right)^{2}}}$ & $p(t)$ [Eq. (10)] & $t_{01}=\int_{\theta_{0}=0}^{\pi / 2} T_{01}\left(\theta_{0}\right) \sin 2 \theta_{0} d \theta_{0}$ \\
\hline$T_{e x}$ & $T_{01}\left(0^{\circ}\right) t / n^{2}$ & $T_{01}\left(0^{\circ}\right) / n^{2}$ & $T_{01}\left(0^{\circ}\right) t / n^{2}$ \\
\hline$r_{1}$ & $r(t)$ [Eq. (11)] & $r(t)$ & $r_{10}$ [Eq. (3)] \\
\hline$r_{2}$ & $r_{10}$ & $r_{10}$ & $r(t)$ \\
\hline
\end{tabular}

The print transmittance is given by Eq. (14), with $T_{i n}, T_{e x}, r_{1}$ and $r_{2}$ being given in the central column of Table 5

$$
T_{1}=\frac{T_{01}\left(0^{\circ}\right)}{n^{2}} p(t) \cdot \frac{\tau}{\left[1-r(t) \rho_{1}\right]\left[1-r_{10} \rho_{2}\right]-r(t) r_{10} \tau^{2}}
$$

The flipped print transmittance is also given by Eq. (14), with $T_{i n}, T_{e x}, r_{1}$ and $r_{2}$ being given in the right column of Table 5 , and with a mutual exchange of the paper internal reflectances $\rho_{1}$ and $\rho_{2}$

$$
T_{2}=\frac{t_{01} T_{01}\left(0^{\circ}\right)}{n^{2}} \cdot t \cdot \frac{\tau}{\left[1-r_{10} \rho_{2}\right]\left[1-r(t) \rho_{1}\right]-r_{10} r(t) \tau^{2}}
$$

According to the orientational bimodal model, the two print transmittance expressions (35) and (36) are different. Although they contain the same most-right fraction, due to the nonpolarity of the multiple reflection-transmission of light inside the print ${ }^{5,13}$, they differ due to the penetration and exit terms $T_{\text {in }}$ and $T_{e x}$. When the ink is in front of the light source, the ink layer is crossed by Lambertian light, whereas when the ink it is in front of the detector, the light captured by the radiance detector crosses the ink at $0^{\circ}$ only.

\section{EXPERIMENTAL TESTING}

We have tested the non-orientational and the orientational bimodal models with solid ink patches printed on white PVC sheets with an offset press. Cyan, magenta and yellow offset inks were almost transparent and did not penetrate into the PVC sheet. The intrinsic parameters of the PVC sheet were obtained using the method presented in Section 3.1.

For each printed solid ink patch, we measured the print reflectance spectrum $R(\lambda)$ using a $45^{\circ} / 0^{\circ}$ geometry and its transmittance $T_{1}(\lambda)$ using a diffuse $/ 0^{\circ}$ geometry. Then, we flipped the patch and measured its transmittance $T_{2}(\lambda)$. For the deduction of the spectral ink transmittance, we first used the non-orientational bimodal model presented in Section 3.2. The invariant ink transmittance $\hat{t}(\lambda)$ is deduced from $R(\lambda)$, from $T_{1}(\lambda)$ and from $T_{2}(\lambda)$ using respectively Eqs. (28), (31) and (33). The three deduced ink transmittance spectra are plotted in Fig. 5. Then, we used the orientational bimodal model presented in Section 3.3. The normal ink transmittance $t(\lambda)$ is deduced again from $R(\lambda)$, from $T_{1}(\lambda)$ and from $T_{2}(\lambda)$ using respectively Eqs. (34), (35) and (36). The deduced ink transmittance spectra are plotted in Fig. 6.

The ink transmittance spectra obtained with the orientational model are very close to each other, except for the short wavelengths where transmittance spectra are noisy, and for the large wavelengths for the cyan ink (see Fig. 6). A possible reason for this difference is the low emission of the light table acting as as Lambertian light source in these wavelength ranges. The match for each ink in the remaining part of the spectrum is excellent. With the Clapper-Yule-like bimodal model, the three deduced ink transmittance spectra are also close to each other, but the matching is less accurate than with the Williams-Clapper model. We can conclude that, for offset inks printed on plastic, in which the inks cannot penetrate, an orientational ink attenuation model (Williams-Clapper model) is preferable to a non-orientational ink attenuation model (Clapper-Yule model). 

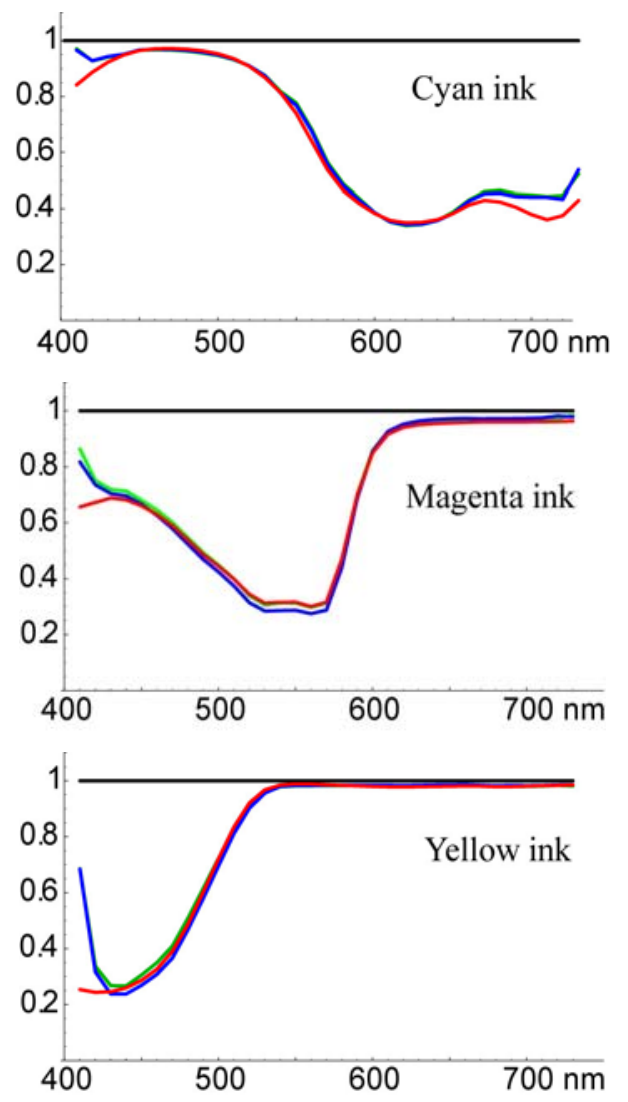

Fig. 5: Invariant ink transmittance spectrum of solid cyan, magenta and yellow inks printed on white PVC, deduced using the non-orientational bimodal model from (a) the measured print reflectance (dotted line), (b) the measured print transmittance with the ink in front of the source (solid line) and (c) the measured print transmittance with the ink in front of the detector (dashed line).
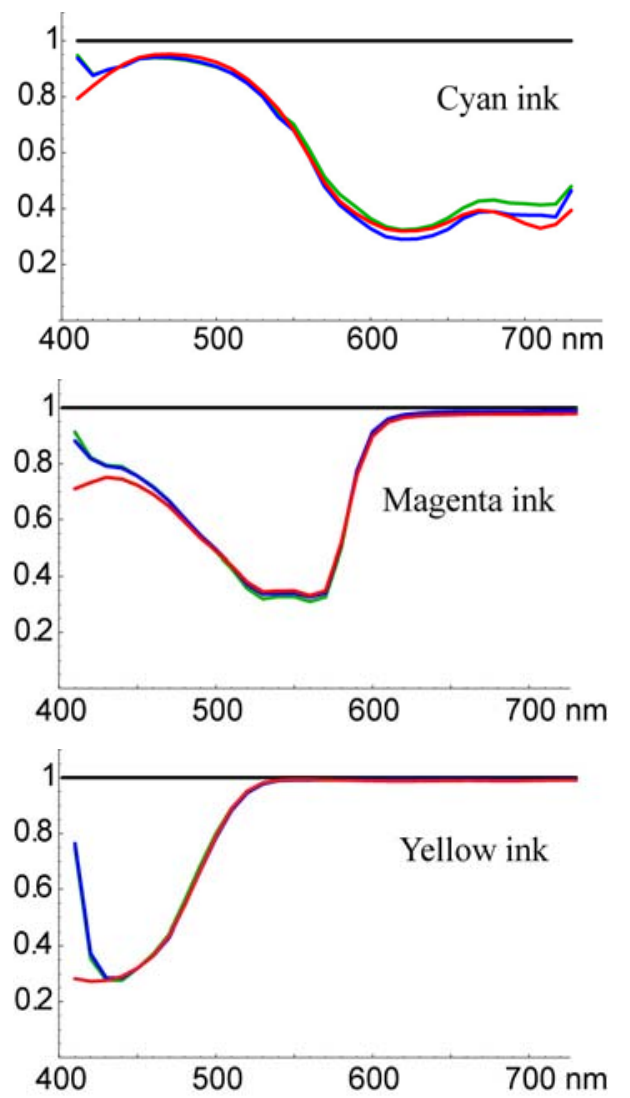

Fig. 6: Normal ink transmittance spectrum of solid cyan, magenta and yellow inks printed on white PVC, deduced using the orientational bimodal model from (a) the measured print reflectance (dotted line), (b) the measured print transmittance with the ink in front of the source (solid line) and (c) the measured print transmittance with the ink in front of the detector (dashed line).

\section{CONLUSIONS}

Reflectance-only models use two spectral parameters: the paper background reflectance and the ink transmittance (called "invariant" in the Clapper-Yule model or "normal" in the Williams-Clapper model). Two reflectance measurements are necessary and sufficient for determining experimentally these two spectral parameters. The paper background reflectance is deduced from the measured paper sheet reflectance, and the ink transmittance is deduced from the measured print reflectance. However, using only reflectance measurements, we have no possibility of verifying the calculated ink transmittance. With the bimodal model, we can use three different measurements performed on a same sample: the reflectance measurement, the transmittance measurement with the ink being at the source side, and the transmittance measurement with the ink being at the detector side. The bimodal model uses four parameters: the paper layer intrinsic parameters (recto reflectance, verso reflectance, and transmittance) and the ink transmittance parameter. The three reflectance and transmittance measurements performed on the unprinted paper sheet allow deducing the three paper intrinsic parameters. Only the ink transmittance parameter remains, which can be deduced from each of the three 
reflectance and transmittance measurements. We can check how close these three computed transmission spectra match. This provides a good practical test for choosing whether the Clapper-Yule model or the Williams-Clapper model is more adapted to the considered type of print. If the three deduced spectra best match with the non-orientational bimodal model, we should use the Clapper-Yule model. On the contrary, if we observe a better match with the orientational bimodal model, we should use the Williams-Clapper model.

\section{AKNOWLEDGEMENT}

The Swiss National Science Foundation gave a partial support for this research under grant 200020-105119.

\section{REFERENCES}

1. F.R. Clapper and J.A.C. Yule, “The Effect of Multiple Internal Reflections on the Densities of Halftone Prints on Paper,” J. Opt. Soc. Am. 43, 600-603 (1953).

2. F. C. Williams and F. R. Clapper, "Multiple Internal Reflections in Photographic Color Prints,” J. Opt. Soc. Am. 29, 595-599 (1953).

3. J.D. Shore and J.P. Spoonhower, "Reflection Density in Photographic Color Prints: Generalizations of the WilliamsClapper Transform,” J. Imaging Sci. Technol. 45, 484-488 (2001).

4. M. Hébert, R.D. Hersch, “Classical Print Reflection Models: A Radiometric Approach”, J. Imaging Sci. Techonol. 48, 363-374 (2004).

5. M. Hébert and R.D. Hersch, “A reflectance and transmittance model for recto-verso halftone prints,” J. Opt. Soc. Am. A 22, 1952-1967 (2006). Available at: http://diwww.epfl.ch/w3lsp/publications/colour/

6. M. Born and E. Wolf, Principle of Optics, Pergamon, Oxford, 7th Edition, 1999.

7. See Ref. 5, Section 2.

8. D. B. Judd, "Fresnel Reflection of Diffusely Incident Light,” J. Res. Natl. Bur. Stand. 29, 329-332 (1942).

9. H.-H. Perkampus, Encyclopedia of Spectroscopy, VCH, 1995.

10. P. Kubelka. "New contributions to the optics of intensely light-scattering material, part ii: Non-homogenous layers". J. Opt. Soc. Am., 44, 330 (1954).

11. J.W. Ryde, The scattering of light by turbid media, Proc. Roy. Soc. (London), A131, 451-464 (1931).

12. G. Kortüm, "Chapter IV. Phenomenological Theories of Absorption and Scattering of Tightly Packed Particles”, Reflectance Spectroscopy, 103-168, Springer Verlag, Berlin, 1969.

13. M. Hébert, Compositional Model for Predicting Multilayer Reflectances and Transmittances in color reproduction, Ph.D. dissertation, p. 91, Ecole Polytechnique Fédérale de Lausanne, 2006. Available at:

http://diwww.epfl.ch/w3lsp/publications/colour/ 